\title{
Impact of lithium releases on ionospheric electron density observed by impedance probe during WIND campaign
}

\author{
Jyunpei Uemoto ${ }^{1}$, Takayuki Ono ${ }^{2}$, Tomohisa Yamada ${ }^{3}$, Tomonori Suzuki ${ }^{2}$, Masa-Yuki Yamamoto ${ }^{3}$, \\ Shigeto Watanabe ${ }^{4}$, Atsushi Kumamoto ${ }^{2}$, and Masahide Iizima ${ }^{5}$ \\ ${ }^{1}$ National Institute of Information and Communications Technology, Tokyo 184-8795, Japan \\ ${ }^{2}$ Graduate School of Science, Tohoku University, Sendai 980-8578, Japan \\ ${ }^{3}$ Graduate School of Engineering, Kochi University of Technology, Kochi 782-8502, Japan \\ ${ }^{4}$ Division of Earth and Planetary Science, Hokkaido University, Sapporo 060-0810, Japan \\ ${ }^{5}$ Daijo Syukutoku Gakuen, Tokyo 174-8645, Japan
}

(Received February 12, 2010; Revised July 1, 2010; Accepted July 2, 2010; Online published August 31, 2010)

\begin{abstract}
This paper presents direct observation of the impact of the lithium releases on the ionospheric electron density during the WIND (wind measurement for ionized and neutral atmospheric dynamics study) campaign conducted on 2 September 2007 in Japan. The direct observation is unique in that the electron density enhancement was observed by using the NEI (number density of electrons by impedance probe) which can measure accurately the absolute value of the electron density, and the distance between the NEI and the LES (lithium ejection system) was very close (several tens of meters). Data analyses of the NEI on-board the sounding rocket S520-23, which was launched from Uchinoura $\left(31.3^{\circ} \mathrm{N}, 131.1^{\circ} \mathrm{E}\right)$ at 19:20 JST (JST $\left.=\mathrm{UT}+9 \mathrm{~h}\right)$, clarifies that lithium releases performed in the descending phase increased the electron density up to approximately $7 \times 10^{5} \mathrm{~cm}^{-3}$. A simple model calculation performed under the assumption that the increased electron density equals the photoionized lithium ion density indicates that the observed electron density enhancements cannot be explained by considering each lithium release as an instantaneous one, but rather by considering a convolution of very short-time intermittent releases. The model calculation is verified by comparison with the observation of the lithium resonance scattering light from the ground.
\end{abstract}

Key words: Ionosphere, impedance probe, electron density, chemical release.

\section{Introduction}

It is important to investigate expansion processes of released materials and its impact on the background physical quantities in space, since chemical releases enable us to observe physical values such as the thermospheric neutral wind and the electric field which are difficult to be observed. This is one of the reasons that the chemical release is a subject which has been extensively studied for many years (e.g., Ma and Schunk, 1991; Schunk and Szuszczewicz, 1991). In the ionosphere, it has been well known that the electron density is dramatically affected by chemical releases (e.g., Lloyd and Haerendel, 1973; Ma and Schunk, 1991; Schunk and Szuszczewicz, 1991; Szuszczewicz et al., 1993). While many sophisticated simulations have been performed to date, some observations of the chemical ion density which accompanies release experiments have been reported to be roughly consistent with simple model calculations (e.g., Hunton, 1993; Szuszczewicz et al., 1996). Hunton (1993) reported that the barium ion density calculated from his model based on a spherically symmetric, collisionless and Gaussian neutral barium shell agreed reasonably well with that observed from the ion spectrome-

Copyright (C) The Society of Geomagnetism and Earth, Planetary and Space Sciences (SGEPSS); The Seismological Society of Japan; The Volcanological Society of Japan; The Geodetic Society of Japan; The Japanese Society for Planetary Sciences; TERRAPUB.

doi:10.5047/eps.2010.07.001 ter (QIMS) on-board the CRESS satellite for the G-12 release experiment. In their experiment, the distance from the barium canister and QIMS was taken to be $4.2 \mathrm{~km}$. Szuszczewicz et al. (1996) reported the in-situ observation of the artificial multi ions during the El Coqui rocket campaign in which the distance from the canisters to the onboard instruments was approximately several hundred meters. They found that the observations generally agreed with a simple spherical free expansion model except for multipeak characteristics due to the ion gyro-motion. The distance from a release point to a sensor is one of the important parameters of the chemical release experiment because the distance concerns what time stage of the expansion process we can observe.

On 2 September 2007, a sounding rocket experiment referred to as the WIND (wind measurement for ionized and neutral atmospheric dynamics study) campaign was conducted in Japan to clarify the interaction process between the ionospheric plasma and the thermospheric neutral wind through a direct observation (Watanabe et al., in preparation). In the WIND campaign, a lithium release experiment was conducted in the descending phase to estimate the thermospheric neutral wind by observing the motion of the released lithium cloud from the ground (Yamamoto et al., in preparation). While the primary purpose of this release experiment is to estimate the thermospheric neutral wind, it provides an opportunity to perform an in-situ observation of 
the impact of the lithium release on the ionospheric electron density. The direct observation during the WIND campaign was unique in terms of the method of the electron density observation and the distance from the sensor to the release point. The electron density enhancement was observed by using the NEI (number density of electrons by impedance probe) developed by Oya (1966) which can measure accurately the absolute value of the electron density, and the distance from the LES (lithium ejection system) to the NEI was very close (several tens of meters). There were no lithium release experiments in which the electron density was observed using the NEI with such a very close distance from the sensor to the release point. The purpose of this paper is to evaluate quantitatively a lithium release impact on the ionospheric electron density enhancement with such a close distance observed during the WIND campaign. In the following sections, we discuss the observed characteristics of the electron density enhancement after summarizing an overview of the lithium release experiment during the WIND campaign, and suggest that the observation can be reasonably explained by considering the convolution of very short-time intermittent releases using a simple spherical free expansion model.

\section{Instruments}

The ionospheric electron density structure is strongly affected by the thermospheric neutral wind through their interaction, such as the ion-drag and the dynamo processes (e.g., Rishbeth, 2000; Heelis, 2004). At night especially, their interaction has been believed to be one of the key factors for controlling the ionospheric irregularities, such as the meso-scale travelling ionospheric disturbance (MSTID) in the mid-latitude ionosphere (e.g., Shiokawa et al., 2003) and the plasma bubble in the equatorial ionosphere (e.g., Abdu, 2001). To evaluate the interaction between the ionosphere and the thermospheric neutral wind through direct observation, the sounding rocket experiment referred to as the WIND campaign was conducted on 2 September 2007 in Japan.

To measure the absolute value of the ionospheric electron density, the NEI was installed on the sounding rocket S-520-23 in the WIND campaign. The specification of the NEI is summarized in Table 1 . The NEI applies RF signals to a probe immersed in plasma and measures an equivalent capacitance $(c(f))$ which depends on the frequency of the RF signals. It has been well known that $c(f)$ becomes minimum at the upper hybrid resonance (UHR) frequency ( $\left.f_{\mathrm{UHR}}\right)$ without depending on an angle between the probe and the magnetic field and a sheath surrounding the probe when plasma is assumed to be collisionless (e.g., Balmain, 1964; Oya and Obayashi, 1966; Suzuki et al., 2010, this issue). $f_{\mathrm{UHR}}$ is a function of the plasma frequency $\left(f_{\mathrm{p}}\right)$ and the cyclotron frequency $\left(f_{\mathrm{c}}\right)$ defined as $f_{\mathrm{UHR}}^{2}=f_{\mathrm{p}}^{2}+f_{\mathrm{c}}^{2}$, thus the electron density can be obtained by substituting $f_{\mathrm{c}}$ calculated from a magnetic field model, such as IGRF. A typical value of the measured $c(f)$ is in the order of $\mathrm{pF}$, thus cancellation of a stray capacity in the electro circuit is important for high accuracy measurement of the electron density. To cancel out the stray capacity, as described in Wakabayashi et al. (2005), the $c(f)$ detection part of
Table 1. Specification for the NEI instrument on-board S-520-23.

\begin{tabular}{ll}
\hline Probe & BeCu, $1.2 \mathrm{~cm} \mathrm{dia.,} \sim 1.0 \mathrm{~m}$ length \\
Frequency Range & $300 \mathrm{kHz}-12 \mathrm{MHz}$ \\
Dynamic Range of Electron Density & $\sim 10^{3}-2 \times 10^{6} \mathrm{el} / \mathrm{cm}^{3}$ \\
Dynamic Range of Equivalent Capacitance & $1-1000 \mathrm{pF}$ \\
Sweep Repetition Period & $500 \mathrm{~ms}$ \\
\hline
\end{tabular}

the NEI consists of a condenser bridge circuit in which a dummy cable is connected to the opposite side of the probe. The NEI installed on the S-520-23 is identical with that installed on the S-310-35 described in Wakabayashi and Ono (2006) except that the electro circuit of the NEI was packaged with the PWM (plasma and wave monitor) in one box. To avoid interference of the local signals between the NEI and the PWM, each local signal was designed to be synchronously swept. The most significant factor for the accuracy of the $f_{\mathrm{UHR}}$ detection is a read out error which depends on a frequency step of the RF signals as described in Wakabayashi and Ono (2006). The frequency step of the NEI on-board S-520-23 was selected to keep the read out error within $4 \%$ for the electron density range above $10^{4}$ $\mathrm{cm}^{-3}$. The NEI probe was installed at $53 \mathrm{~cm}$ from the rocket apex. Then, in the ascending phase when the rocket velocity vector and the spin axis are almost parallel, the wake of the rocket body does not significantly influence the electron density measurement, while in the descending phase, it influences the measurement synchronizing with a rocket spin rate of approximately $1 \mathrm{~Hz}$ (see Fig. 3).

The LES consists of three canisters with each canister containing solid lithium of $130 \mathrm{~g}$. Each canister was designed to gasify and release the lithium individually by thermite process within $10 \pm 5 \mathrm{~s}$ at approximately $10 \mathrm{~s}$ after receiving an ejection signal. The released lithium resonance scattering light of a $670.8 \mathrm{~nm}$ wavelength was observed using CCD imagers synchronously from Shionomisaki $\left(33.5^{\circ} \mathrm{N}, 135.8^{\circ} \mathrm{E}\right)$, Miyazaki $\left(31.8^{\circ} \mathrm{N}, 131.4^{\circ} \mathrm{E}\right)$, Uchinoura $\left(31.3^{\circ} \mathrm{N}, 131.1^{\circ} \mathrm{E}\right)$ and Amami $\left(28.5^{\circ} \mathrm{N}, 129.7^{\circ} \mathrm{E}\right)$. The sampling rate and exposure time are $8 \mathrm{~s}$ and $4 \mathrm{~s}$ for the time period from $373 \mathrm{~s}$ to $478 \mathrm{~s}$ after the launch, $20 \mathrm{~s}$ and $15 \mathrm{~s}$ for the period from $494 \mathrm{~s}$ to $1229 \mathrm{~s}$, and $60 \mathrm{~s}$ and $30 \mathrm{~s}$ for the period from $1299 \mathrm{~s}$ and $3344 \mathrm{~s}$, respectively. The details of the LES system, ground observation of the lithium resonance scattering light and the other on-board instruments are provided in Yamamoto et al. (in preparation) and Watanabe et al. (in preparation).

\section{NEI Observation}

The sounding rocket was launched from Uchinoura at 19:20 JST (JST $=$ UT +9 h) on 2 September 2007 and reached its apex altitude of $279.4 \mathrm{~km}$ at $268 \mathrm{~s}$ after the launch (Fig. 1). The probe of the NEI was extended at the altitude of $81.8 \mathrm{~km}$ at $56.5 \mathrm{~s}$ after the launch, and the NEI successfully measured $c(f)$ during the rocket flight after the probe extension. The LES was separated from the rocket body on which the NEI was installed around the apex altitude at $358 \mathrm{~s}$ after the launch with the separation velocity of approximately $0.5 \mathrm{~m} / \mathrm{s}$. After the separation, the lithium 


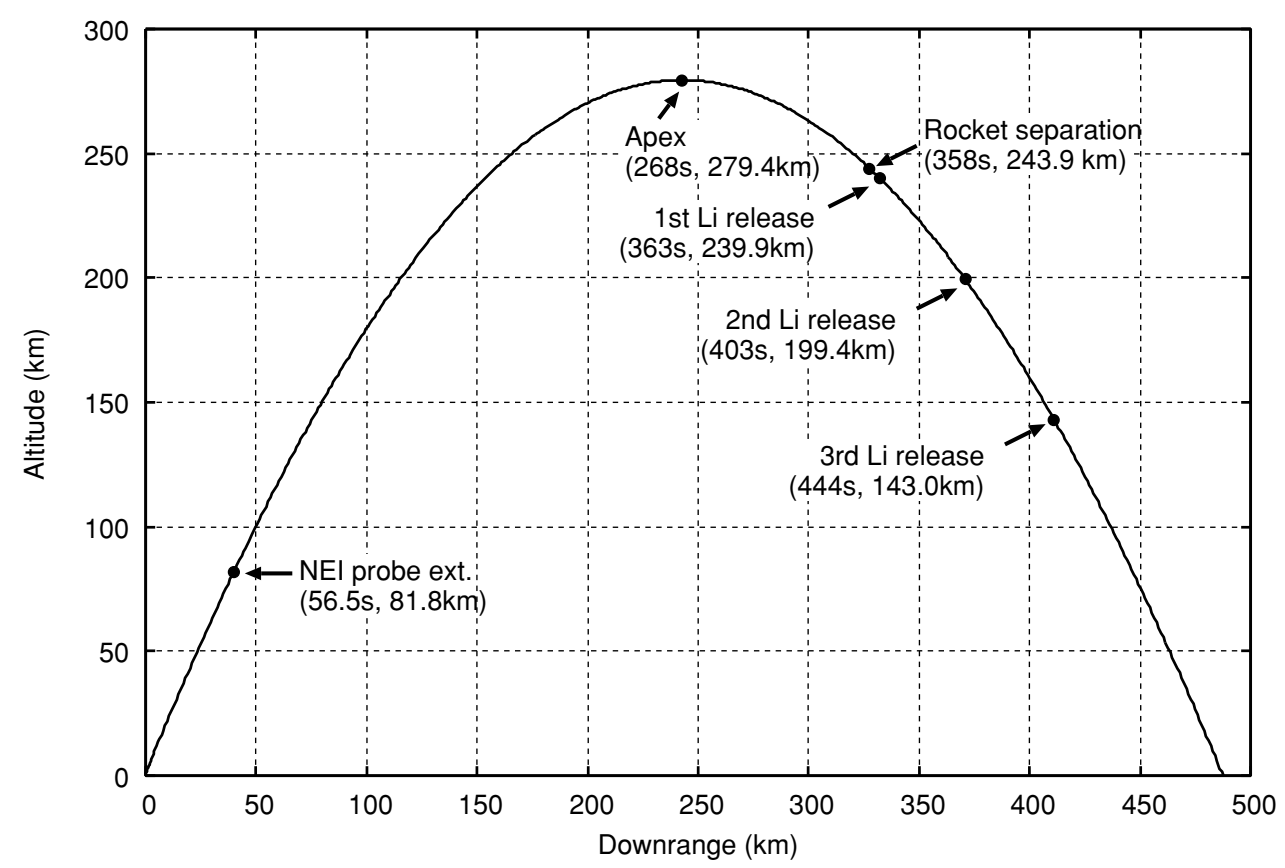

Fig. 1. Trajectory of the sounding rocket, S-520-23 with the flight events related to the NEI observation and lithium releases. It should be noted that each lithium release in the panel is just a scheduled one, and that there were some differences between the scheduled and actual releases.

release experiment was conducted. As shown in Fig. 1, three lithium releases were scheduled to start at $363 \mathrm{~s}, 403 \mathrm{~s}$ and $444 \mathrm{~s}$ after the launch. Hereinafter, these releases are referred to as L1, L2 and L3 in this paper, respectively. The distances between the NEI and the LES for L1, L2 and L3 are estimated from the separation velocity as approximately $7.5 \mathrm{~m}, 27.5 \mathrm{~m}$ and $48.0 \mathrm{~m}$.

Figure 2 shows the examples of the measured $c(f)$ curves. Figure 2(a)-(d) (left panels) shows the measured $c(f)$ curves in the ascending phase, while Fig. 2(e)-(h) (right panels) shows them in the descending phase. These panels are arranged as each pair of $c(f)$ curves in a row was obtained at approximately the same altitude. Before L1, the $c(f)$ curves are not disturbed around $f_{\mathrm{UHR}}$, and $f_{\mathrm{UHR}}$ can be accurately identified as noted by the arrows in Fig. 2(a)(e), while after $\mathrm{L} 1$, the $c(f)$ curves are heavily disturbed, and many $c(f)$ curves from which $f_{\mathrm{UHR}}$ cannot be accurately identified were obtained. Figure $2(\mathrm{~g})$ is an example of the heavily disturbed $c(f)$ curves. As shown in Fig. 2(g), severe noises with a large amplitude are superimposed on the $c(f)$ curve around $f_{\mathrm{UHR}}$ preventing the determination of $f_{\text {UHR }}$ accurately. It should be noted, however, that $f_{\text {UHR }}$ can be seen around the frequency of $3.5 \mathrm{MHz}$ in Fig. 2(g), and this frequency is clearly higher than that in Fig. 2(c). On the other hand, some $c(f)$ curves from which $f_{\mathrm{UHR}}$ can be accurately identified were obtained even after L1 as shown in Fig. 2(f) and 2(h). $f_{\mathrm{UHR}}$ shown in Fig. 2(f) and 2(h) is also clearly higher than that shown in Fig. 2(b) and 2(d), respectively. These facts imply that the lithium releases increased the electron density.

Figure 3 shows the altitude profiles of the electron density deduced from $f_{\mathrm{UHR}}$ in the ascending (left panel) and descending (right panel) phases which depict the whole picture of the electron density enhancement in the descending phase. Each opened red circle with an alphabetical notation corresponds to the altitude at which each $c(f)$ curve shown in Fig. 2 was obtained. It should be noted that the electron density profile in the descending phase is composed of only the $c(f)$ curves, such as those shown in Fig. 2(f) and 2(h) in which $f_{\mathrm{UHR}}$ can be accurately identified. In other words, the heavily disturbed $c(f)$ curves, such as those shown in Fig. 2(g), were excluded. In the altitude range above approximately $240 \mathrm{~km}$, the electron density profile in the descending phase is periodically modulated, and its amplitude is larger than that in the ascending phase. This modulation is due to the wake effect of the rocket body mentioned in Section 2. On the other hand, it can be noted that the local peaks of very high electron density are seen in the altitude range below $240 \mathrm{~km}$ in the descending phase. These peaks are not due to the wake effect because the electron density is extremely high compared with that in the ascending phase.

In the following section, we suggest that these observed electron density peaks are caused by the lithium releases by performing a simple model calculation and by comparison with the luminosity profile obtained from the ground observation of the lithium resonance scattering light.

\section{Analysis and Discussion \\ 4.1 Deduction of electron density variation}

At first, we deduced the increased value of the electron density caused by the lithium releases $\left(\Delta n_{\mathrm{e}}\right)$ based on the assumption that the electron density in the descending phase would be same as that in the ascending phase if the lithium release experiment had not been conducted. This assumption appears to be valid since both the electron density profiles in the ascending and descending phases are almost identical in the altitude regions above $240 \mathrm{~km}$ where the electron density was obtained before starting the lithium releases and below $120 \mathrm{~km}$ where the lithium releases seemed not to have affected the electron density. Based on this as- 

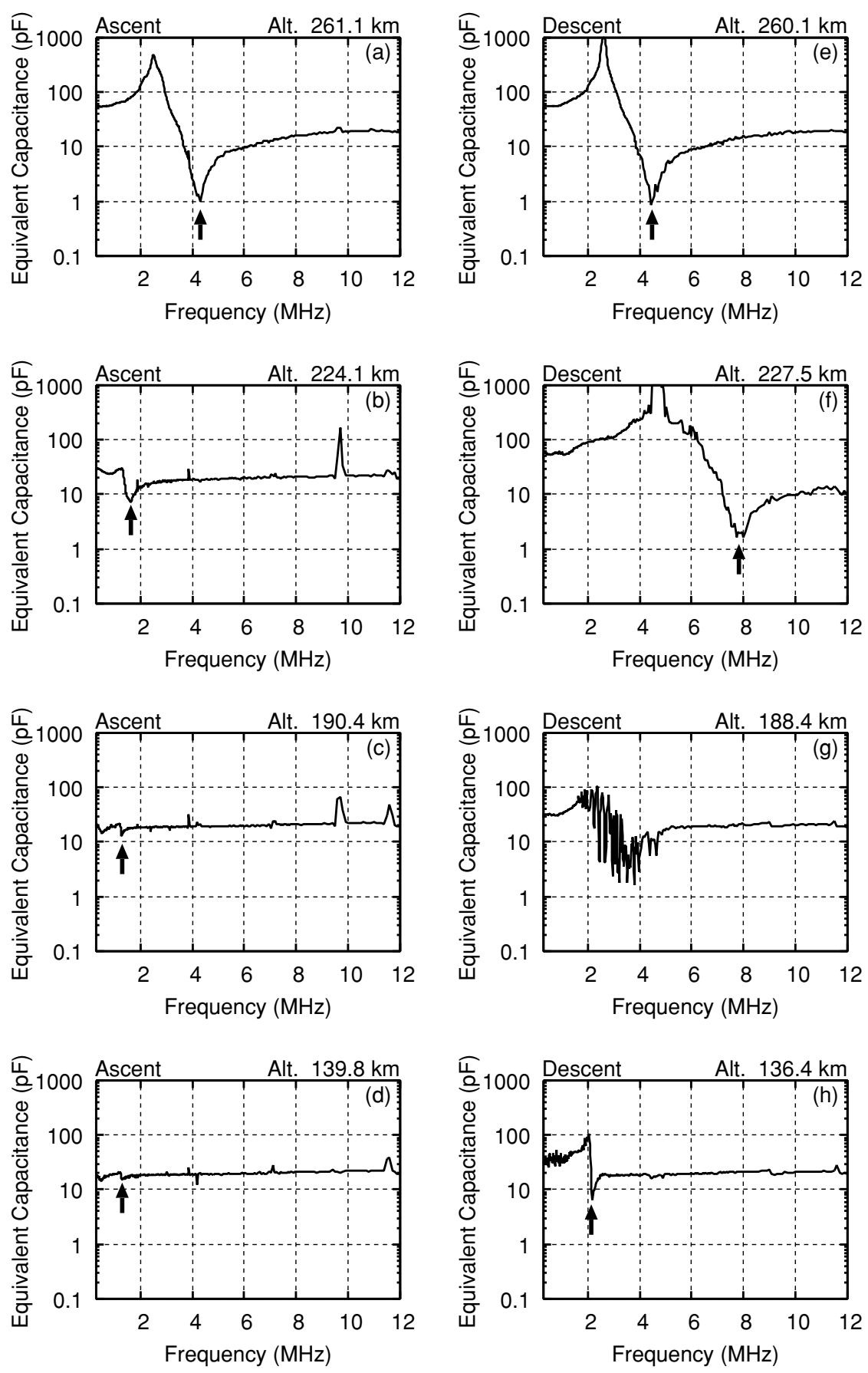

Fig. 2. Examples of the $c(f)$ curves obtained using the NEI during the ascending (left panels) and descending (right panels) phases. Left and right panels in each row are the $c(f)$ curves obtained at approximately the same altitude. Each arrow in each panel indicates the frequency of $f_{\mathrm{UHR}}$. The $c(f)$ curves of the panels $(\mathrm{f}),(\mathrm{g})$ and $(\mathrm{h})$ were obtained after starting the lithium release experiment.

sumption, we subtracted the electron density in the ascending phase from that in the descending phase after we estimated the electron density in the ascending phase at the altitudes where the electron density in the descending phase were sampled by performing the liner interpolation. Before we consider the difference of the electron density between the ascending and descending phases as $\Delta n_{\mathrm{e}}$ caused by the lithium releases, the wake effect should be eliminated because the amplitude of the electron density modulation caused by the wake effect is proportional to the background electron density. In fact, the modulation caused by the wake effect above the $240 \mathrm{~km}$ altitude is comparable to the elec- tron density enhancement around the $140 \mathrm{~km}$ altitude which appears to be related to L3. To eliminate the wake effect, we calculated the ratio of the electron density profiles during the descending to ascending phases (Fig. 4(a)). As seen in Fig. 4(a), the ratio is small in the altitude region above $240 \mathrm{~km}$ where the wake significantly affects the electron density, while it becomes large after the lithium releases. In this paper, we regard the electron density enhancement with a ratio of larger than 0.8 to be caused by the lithium releases. Figure 4(b) shows the electron density enhancement $\Delta n_{\mathrm{e}}$ caused by the lithium releases from which the wake effect was eliminated. 

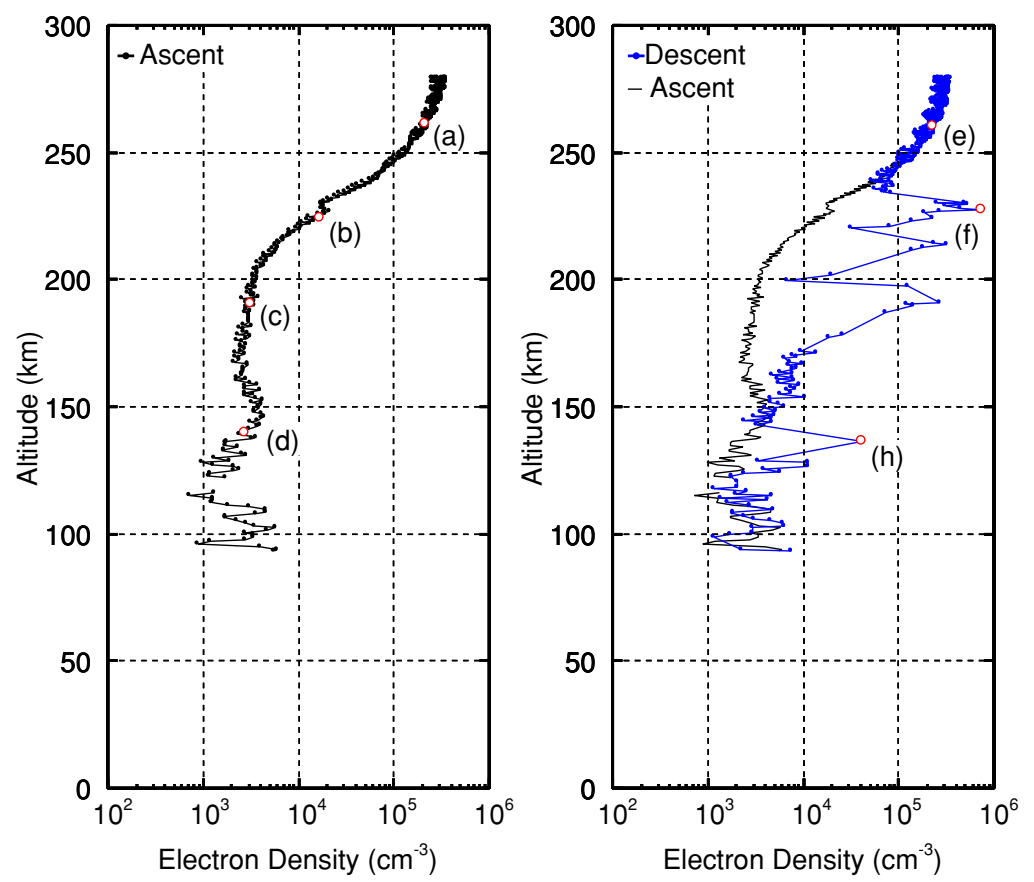

Fig. 3. Altitude profiles of the electron density derived from $f_{\text {UHR }}$ observed by the NEI during the ascending (left panel) and descending (right panel) phases. In the right panel, the altitude profile in the ascending phase is superimposed as a black thin line. Each red opened circle with the alphabetical notation indicates the altitude at which each $c(f)$ curve shown in Fig. 2 was observed.
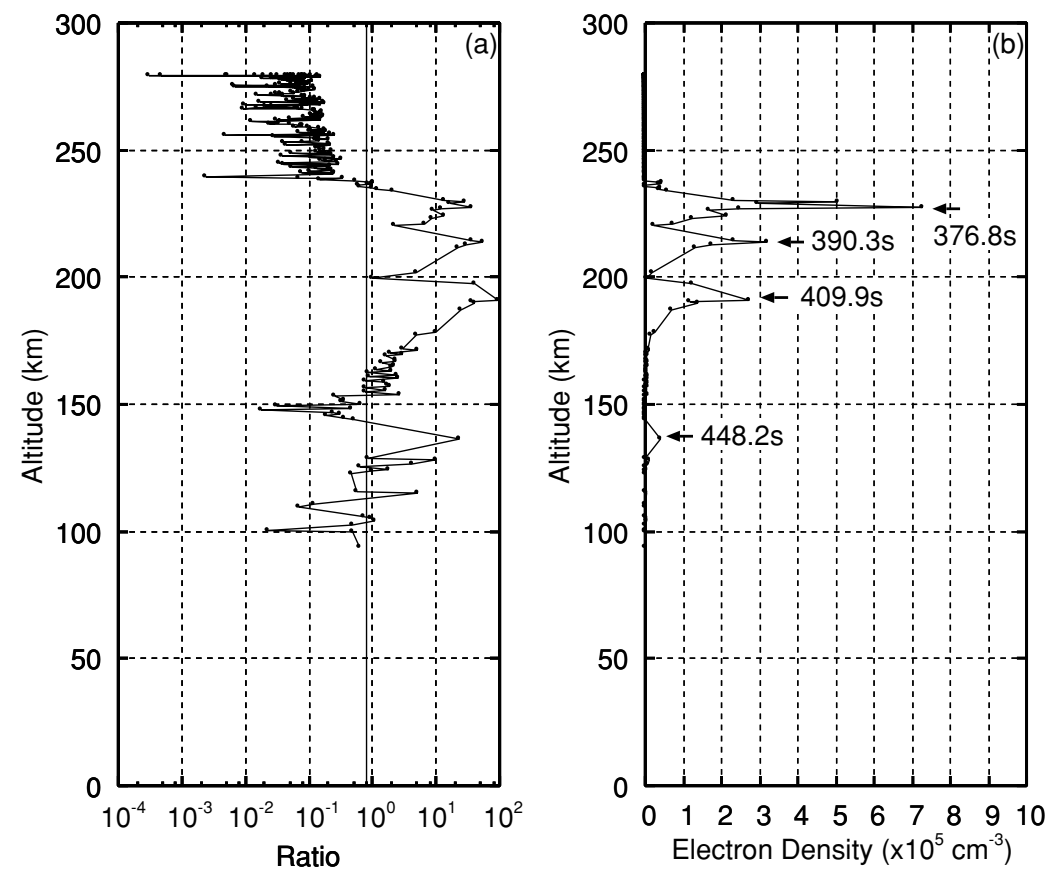

Fig. 4. Ratio of the electron density profiles during the descending to ascending phases (a) and the altitude profile of the electron density variation without the wake effect (b). The vertical solid line in the left panel corresponds to the threshold which divides the lithium releases effect on the electron density from the wake one. The time after launch of the major density enhancement peaks is inserted in the right panel.

Figure 4(b) indicates that the lithium releases increased the electron density up to approximately $7 \times 10^{5} \mathrm{~cm}^{-3}$, and each enhancement lasts approximately 10 to $20 \mathrm{~s}$. The electron density peaks are almost synchronized with L1, L2 and L3, however, the density peak at around $210 \mathrm{~km}$ is not synchronized with any scheduled lithium releases. Although Szuszczewicz et al. (1996) reported that a single chemical release can generate plural peaks depending on gyro-motion periods of ions, the density peak at around $210 \mathrm{~km}$ is not due to the gyro-motion effect because it was observed approximately $10 \mathrm{~s}$ after L1, and the gyro-motion periods of ions are less than $1 \mathrm{~s}$. The density peak at around $210 \mathrm{~km}$ appears to imply that an unexpected lithium release occurred. 


\subsection{Estimation of altitudinal lithium releases profile}

Figure 5(a) shows a picture of the lithium resonance scattering light taken from Uchinoura at $454 \mathrm{~s}$ after the launch. To compare the distribution of the lithium resonance scattering light shown in Fig. 5(a) with the electron density enhancement, it is required to deduce an altitudinal luminosity profile along the rocket trajectory. To deduce the altitudinal profile, the lithium resonance scattering light shown in Fig. 5(a) was horizontally sliced. Then, the horizontally sliced profiles were individually fitted with the Gaussian function. Although these profiles consist of the integrated luminosity along the line-of-sight, the peak value of each fitted Gaussian function can be considered as a good proxy of the true luminosity at each altitude. Thus, we adopted the peak values of the Gaussian functions arranged according to altitude as the altitudinal luminosity profile along the rocket trajectory. Figure 5(b) shows the altitudinal luminosity profile along the rocket trajectory deduced by the procedure described above. Although it is almost impossible to identify any signs of L3 shown in Fig. 5(a), a weak luminosity enhancement (or modulation) can be confirmed around the altitude of $140 \mathrm{~km}$ as shown in Fig. 5(b) which indicates that L3 was conducted as scheduled. In the altitude range above $170 \mathrm{~km}$, the luminosity profile appears to have been formed by combining two or more releases. Although it is then difficult to decompose the profile into each lithium release from Fig. 5(b), on the other hand, we confirmed, by analyzing a sequence of the pictures with the sampling rate of $8 \mathrm{~s}$, that intense releases occurred at the altitudes noted by the arrows shown in Fig. 5(b). These altitudes agree well with the scheduled ones, and then they can be regarded as L1 and L2. In this context, the luminosity profiles above and below the altitudes noted by the arrows are inferred to be formed by L1 and L2, respectively. The estimated luminosity profiles originating from L1 and L2 are shown as red curves in Fig. 5(b). To deduce these curves, the Gaussian functions were fitted to the luminosity profiles above and below the altitudes noted by arrows, respectively. It can be noted that there is a large difference between the black and red curves in the altitude range around $210 \mathrm{~km}$. The value of the difference is shown as the blue curve in Fig. 5(b). The shape of blue curve also resembles the Gaussian function. This resemblance indicates that an unexpected lithium release occurred at around the altitude of $210 \mathrm{~km}$. It should be noted here that vertical expansions of lithium would be slightly different in expanding directions since the LES was moving downward along the rocket trajectory and the atmospheric density naturally decreases with height. Actually, we confirmed that the gradient of the upward expansion of L1 differed from that of the downward expansion of L2, even after consideration of each elapsed time from the release. However, it can be noted that the difference is sufficiently small for deducing the occurrence of the unexpected lithium release. Therefore, in the fitting processes for two luminosity profiles of L1 and L2, we used the simple Gaussian function with neglecting the relatively small difference between upward and downward expansions.

\subsection{Model calculation and comparison}

Both the analysis results of the electron density and luminosity profiles indicate that the unexpected lithium re-

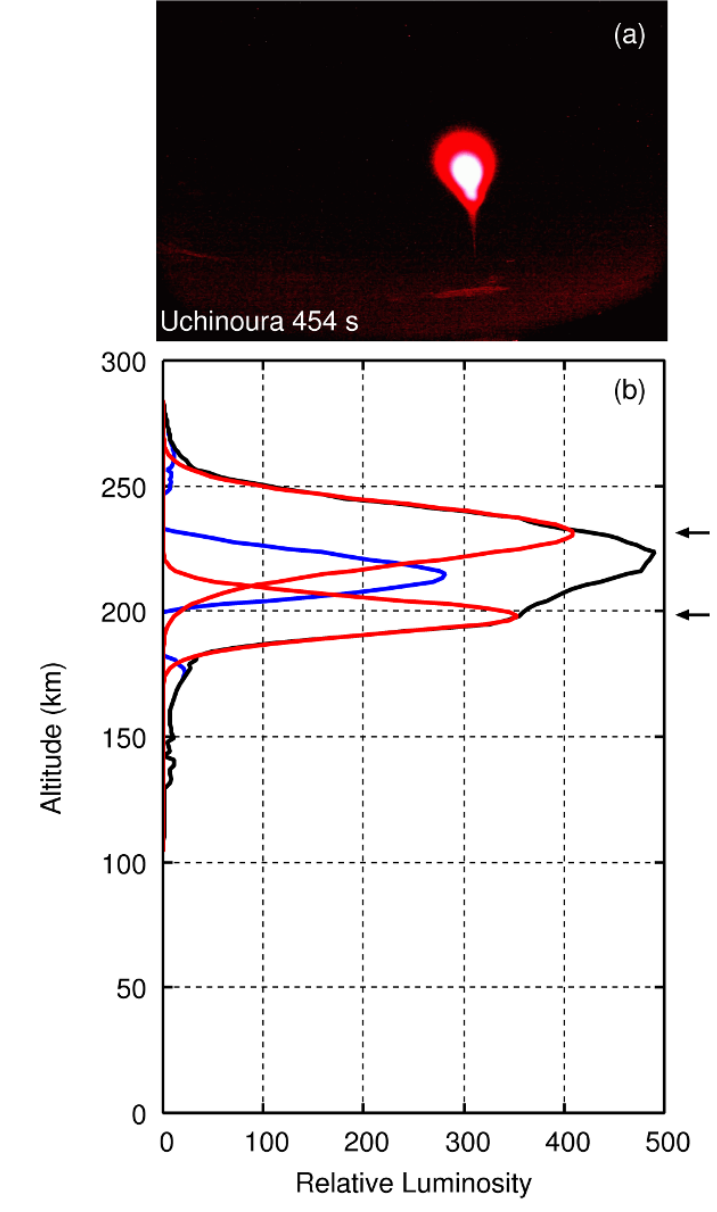

Fig. 5. Lithium resonance scattering light observed at Uchinoura at $454 \mathrm{~s}$ after the launch (a) and luminosity profile along the rocket trajectory (b). The brightness and contrast of the panel (a) are arranged for easier recognition. Black, red and blue lines in the panel (b) indicate the whole luminosity, Gaussian fitting curves for L1 and L2, and difference between the whole luminosity and that of L1 and L2, respectively.

lease occurred between L1 and L2. Hereinafter, we refer to this unexpected lithium release as L1.5. The existence of L1.5 prevents us from investigating the relationship between the electron density enhancement and the lithium releases based on the scheduled time sequence of the lithium releases. Instead, we calculate an altitude profile of the vaporized lithium from the electron density enhancement $\Delta n_{\mathrm{e}}$ using a simple lithium expansion model under the assumption that $\Delta n_{\mathrm{e}}$ equals the photoionized lithium density. By comparing an altitude profile of the vaporized lithium calculated from the model described below and that of the luminosity shown in Fig. 5(b), we suggest that $\Delta n_{\mathrm{e}}$ can be reasonably explained by considering a convolution of very short-time intermittent releases.

We assume that the vaporized lithium for a single lithium release is spherically distributed, and its density is expressed as $n(r, t)$, where $r$ and $t$ are the distance from the release point (or from the lithium cloud center) and the elapsed time from the release, respectively. Although Bernhardt (1992) reported that the lithium cloud becomes a shell-like structure within several tens of seconds after the release, it has not been well known whether the lithium cloud becomes a shell-like structure within a very short time 


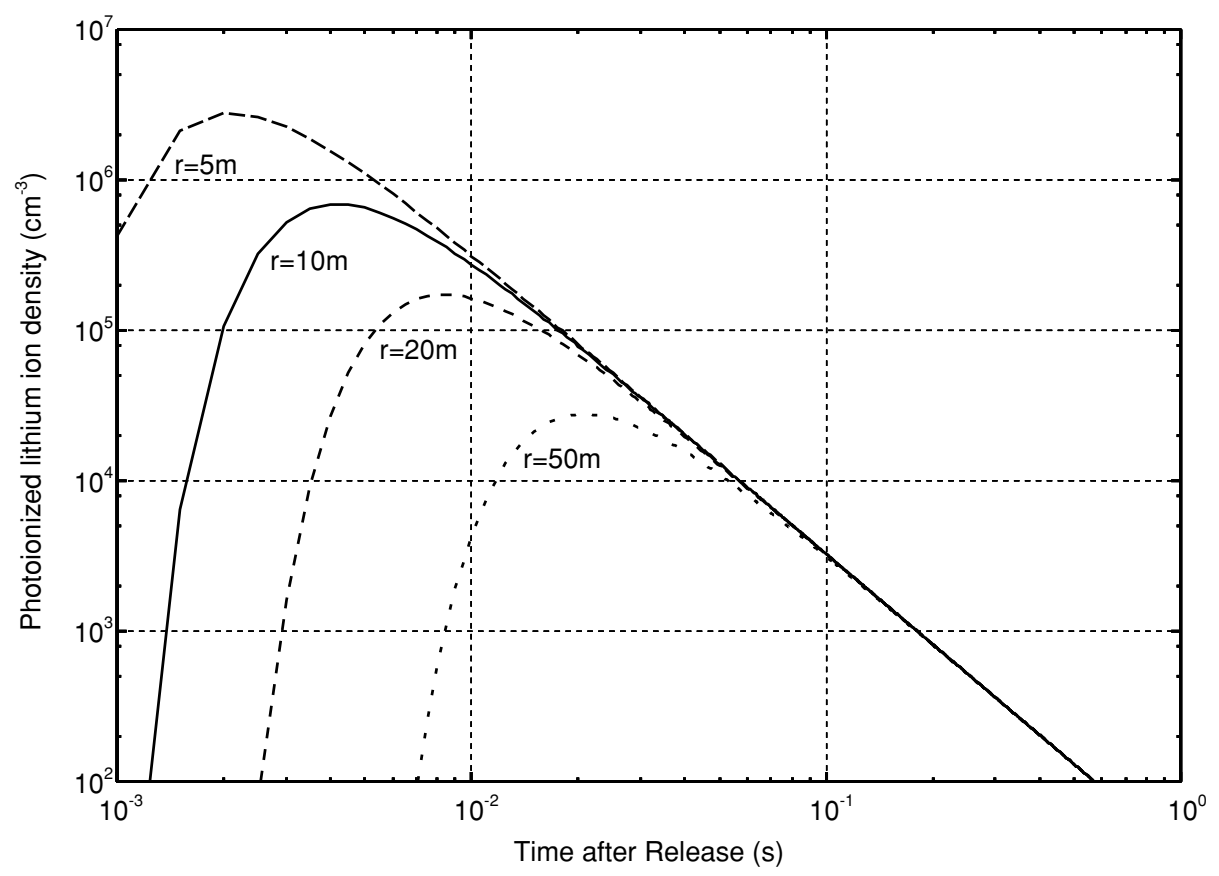

Fig. 6. Calculated time variations of the photoionized lithium ion density at the distances of 5, 10, 20 and $50 \mathrm{~m}$ from the cloud center for a single lithium release with the amount of $0.1 \mathrm{~g}$.

( $<1 \mathrm{~s}$ ) from the release as pointed out by Szuszczewicz et al. (1996). We assume that the lithium cloud expands through the free expansion process, although at later times the cloud is thought to expand through the diffusive expansion process (e.g., Bernhardt, 1979). In this context, we adopt a spherical free expansion model and define $n(r, t)$ by

$$
n(r, t)=\frac{N_{0}(T)}{\pi^{3 / 2}\left(v_{\mathrm{th}} t\right)^{3}} \exp \left[-\frac{r^{2}}{\left(v_{\mathrm{th}} t\right)^{2}}\right],
$$

where $T, N_{0}(T)$ and $v_{\text {th }}$ are the release time, the amount of vaporized lithium at $T$ and the lithium thermal velocity, respectively. The released lithium is assumed to be vaporized instantaneously in this paper. $N_{0}(T)$ in Eq. (1) is defined as

$$
N_{0}(T)=x(T) / M
$$

where $x(T)$ is the amount of vaporized lithium at $T$, and $M$ is the mass of a lithium atom. Equation (1) is almost identical to that of the spherical free expansion model adopted in Bernhardt (1979) and Szuszczewicz et al. (1996), and this equation indicates that $n(r, t)$ is expressed as the Gaussian distribution, and it decreases monotonically in proportion to $t^{-3}$.

The vaporized lithium is assumed to be ionized through the photoionization process:

$$
n_{\mathrm{Li}^{+}}(r, t)=n(r, t)\left[1-\exp \left(-\frac{t}{\tau_{\mathrm{Li}}}\right)\right],
$$

where $n_{\mathrm{Li}^{+}}(r, t)$ is the lithium ion density, and $\tau_{\mathrm{Li}}$ is the lithium ionization constant. Equation (3) is adopted from Szuszczewicz et al. (1996). Substituting Eqs. (1) and (2) into Eq. (3) gives

$$
n_{\mathrm{Li}^{+}}(r, t)=x(T) \frac{1}{M \pi^{3 / 2}\left(v_{\mathrm{th}} t\right)^{3}} \exp \left[-\frac{r^{2}}{\left(v_{\mathrm{th}} t\right)^{2}}\right]
$$

$$
\begin{aligned}
& \cdot\left[1-\exp \left(-\frac{t}{\tau_{\mathrm{Li}}}\right)\right] \\
= & x(T) g(r, t) .
\end{aligned}
$$

In Eq. (4), we defined $g(r, t)$ as the right-hand side term except for $x(T)$. In this paper, $v_{\text {th }}$ is $2400 \mathrm{~m} / \mathrm{s}$ which is the thermal velocity at the lithium vaporization temperature of $1600 \mathrm{~K}$, and $\tau_{\mathrm{Li}}$ is $3450 \mathrm{~s}$. We assumed $v_{\text {th }}$ to be constant as done by Szuszczewicz et al. (1996).

Figure 6 shows time variations of the photoionized lithium ion density at $r=5,10,20$ and $50 \mathrm{~m}$ calculated from Eq. (4) when the lithium of $0.1 \mathrm{~g}$ is vaporized at $T=0$. For example, the lithium ion density at $r=10 \mathrm{~m}$ (solid line) is maximum approximately at $10^{6} \mathrm{~cm}^{-3}$ at $4 \mathrm{~ms}$ after the release, and it becomes less than $10^{2} \mathrm{~cm}^{-3}$ within $1 \mathrm{~s}$ after the release. This result implies that the released lithium contributes to the observed $\Delta n_{\mathrm{e}}$ shown in Fig. 4(b) through the photoionization process within only a short time period $(<1 \mathrm{~s})$ after the release. This is why the diffusive expansion is ignored in this paper. This result also points out that the time resolution of the NEI (approximately $500 \mathrm{~ms}$ ) is insufficient to observe the time variation of the photoionized lithium ion density. This shortage of the time resolution forces performance of the linear interpolation between the observed electron density values. On the other hand, as mentioned in Section 4.1, each electron density enhancement shown in Fig. 4(b) lasts approximately 10 to $20 \mathrm{~s}$. Referring to the time variations of the photoionized lithium ion density shown in Fig. 6, it is noted that a single instantaneous lithium release cannot explain the observed $\Delta n_{\mathrm{e}}$ profile because the photoionized lithium ion density dramatically decreases within $1 \mathrm{~s}$ after the release. Then, the lithium ion density is assumed to be expressed by a con- 
volution of intermittent releases:

$$
n_{\mathrm{Li}^{+}}(r, t)=\sum_{\tau=0}^{t} x(\tau) g(r, t-\tau) .
$$

Equation (5) is a convolutional sum. When the calculation time step is defined as $\Delta t$, the photoionized lithium ion density at $\Delta t$ after the time of release $t^{\prime}$ is

$$
n_{\mathrm{Li}^{+}}\left(r, t=t^{\prime}+\Delta t\right)=x\left(t^{\prime}\right) g(r, \Delta t)+x\left(t^{\prime}+\Delta t\right) g(r, 0) \text {. }
$$

In Eq. (6), $g(r, 0)$ on the second term of the right-hand side is zero, then $x\left(t^{\prime}\right)$ can be obtained:

$$
x\left(t^{\prime}\right)=n_{\mathrm{Li}^{+}}\left(r, t=t^{\prime}+\Delta t\right) / g(r, \Delta t) .
$$

Similarly, the photoionized lithium ion density at the next time step $\left(t=t^{\prime}+2 \Delta t\right)$ is

$$
\begin{aligned}
n_{\mathrm{Li}^{+}}\left(r, t=t^{\prime}+2 \Delta t\right)= & x\left(t^{\prime}\right) g(r, 2 \Delta t)+x\left(t^{\prime}+\Delta t\right) g(r, \Delta t) \\
& +x\left(t^{\prime}+2 \Delta t\right) g(r, 0) .
\end{aligned}
$$

In Eq. (8), the unknown factor is only $x\left(t^{\prime}+\Delta t\right)$ because $g(r, 0)$ is zero and $x\left(t^{\prime}\right)$ is already given by Eq. (7). Then $x\left(t^{\prime}+\Delta t\right)$ can be calculated from Eq. (8). By performing similar calculations in later time steps, the time variation of the photoionized lithium ion density can be deduced. The total amount of the vaporized lithium is

$$
X_{\text {tot }}=\sum_{\tau} x(\tau)
$$

As expressed in Eqs. (7) and (8), the photoionized lithium ion density is divided by $g(r, \Delta t)$ to obtain the amount of vaporized lithium at each time step. This indicates that the calculation would fail when $g(r, \Delta t)$ is extremely small. The value of $g(r, \Delta t)$ depends on the time step as well as the distance from the cloud center as seen in Fig. 6. To avoid the problem due to $g(r, \Delta t)$, we performed a model calculation under the assumption that the distance from the cloud center is constant $(r=10 \mathrm{~m}$ ), although this assumption is over simplified because the NEI moved away from the LES with the speed of $0.5 \mathrm{~m} / \mathrm{s}$ after the separation as mentioned in Section 3.

The amount of the vaporized lithium calculated from Eq. (5) with the time step of $4 \mathrm{~ms}$ is shown in Fig. 7. It should be noted that the calculated time variation of the vaporized lithium amount was arranged along the altitude by using the trajectory data to more easily compare the calculation with the observations. It should be noted that the time variations of vaporized lithium and $\Delta n_{\mathrm{e}}$ are almost identical by comparing Figs. 7 and 4(b). This indicates that only the vaporized lithium within a short time period $(<1 \mathrm{~s})$ after the release can contribute to $\Delta n_{\mathrm{e}}$. On the other hand, even when the maximum $\Delta n_{\mathrm{e}}$ was observed, the amount of vaporized lithium was estimated as $0.04 \mathrm{~g}$. This result indicates that the convolution effect of plural intermittent releases expressed in Eq. (5) is important in the short time period $(<1 \mathrm{~s})$ because the required amount for increasing the electron density up to $7 \times 10^{5} \mathrm{~cm}^{-3}$ at $r=10 \mathrm{~m}$ is more than $0.1 \mathrm{~g}$ when a single release is considered (see Fig. 6). The

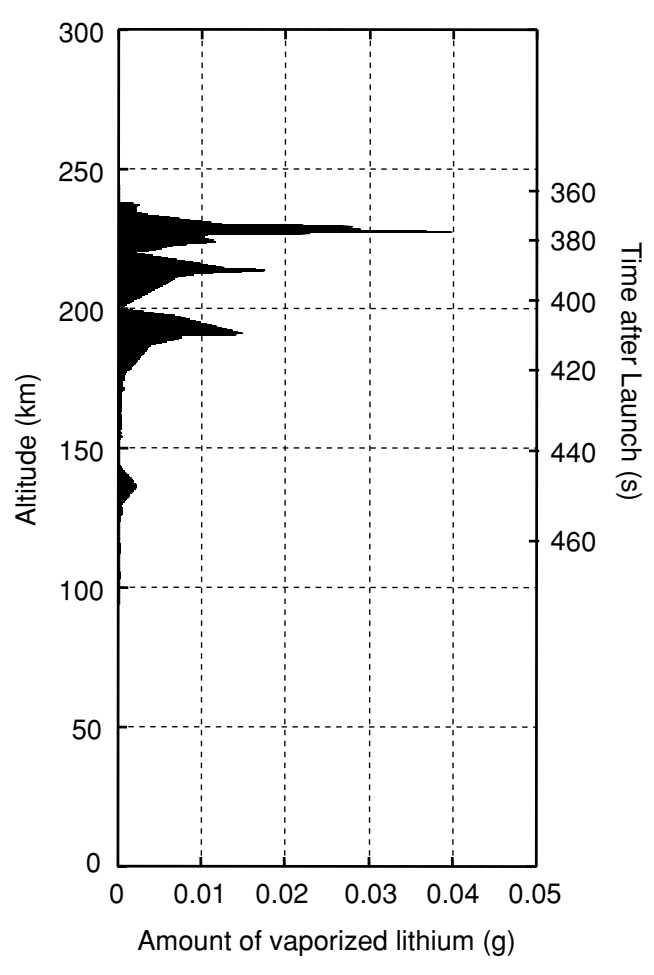

Fig. 7. Calculated profile of the vaporized lithium amount. The left-hand and right-hand vertical axes are altitude and time after launch, respectively.

total amount of vaporized lithium calculated from Eq. (9) is approximately $99 \mathrm{~g}$. This amount corresponds to approximately $25 \%$ of $390 \mathrm{~g}$ which is the total amount of the filled lithium in the canisters. (Szuszczewicz et al. (1996) assumed the effective vaporization level as $40 \%$.) It should be noted that a direct comparison cannot be strictly performed between the calculated profile with the luminosity profile. This is because the observed lithium resonance scattering light from the ground is a time dependent column value along the line of sight. However, it can be pointed out by comparing Figs. 7 and 5(b) that the peak altitudes of the vaporized lithium estimated from $\Delta n_{\mathrm{e}}$ agree well with those estimated from the ground observation. This good agreement verifies the model calculation and indicates that the electron density enhancement observed using the NEI during the WIND campaign can be explained by considering a convolution of very short-time intermittent releases with the simple spherical free expansion model. Finally, it should be mentioned that the model calculation provided here is oversimplified based on the following points of view: (1) the time variation of the distance between the LES and the NEI, (2) the optical thickness of the lithium cloud, and (3) the altitudinal variation of the photoionization rate due to the solar zenith angle change are not included. Although the assumptions (1) and (2) affect the estimated total amount and the altitude profile of the vaporized lithium in some degree, these assumptions do not deny the result that the observed electron density enhancement can be explained by considering a convolution of very short-time intermittent releases. On the other hand, the assumption (3) is inadequate for $\Delta n_{\mathrm{e}}$ around $445 \mathrm{~s}$ after the launch because the sun light did not 
reach the altitude region directly where the rocket passed through at $445 \mathrm{~s}$ after the launch. Nevertheless, although it is very weak, the lithium resonance scattering light around this altitude region was observed from the ground as shown in Fig. 5(b). This fact indicates that the sun light reached this altitude region from somewhere. Clarifying the mechanism of the electron density enhancement at around $445 \mathrm{~s}$ after the launch is a subject which remains to be investigated further.

\section{Conclusion}

To evaluate quantitatively the lithium release impact on the ionospheric density enhancement during the WIND campaign, we analyzed the $c(f)$ data obtained from the NEI which can measure accurately the absolute value of the electron density. It was clarified that the lithium releases performed in the descending phase increased the electron density up to approximately $7 \times 10^{5} \mathrm{~cm}^{-3}$. This is a first observational result showing the impact of the lithium releases on the ionospheric electron density at the very close distance of several ten meters from the sensor to the release point. To investigate the relationship between the observed electron density enhancement and the lithium releases, we developed a convolution model of the simple free expansion. By using this convolution model, we found that the peak altitudes of the vaporized lithium estimated from the electron density enhancement agreed well with those estimated from the ground observation of the lithium resonance scattering light. Based on this good agreement, it is suggested that the free expansion model is effective at the distance of several ten meters from the release point and the effect of superposition of releases cannot be ignored. On the other hand, it was found that the electron density was increased at the altitude range where the sun light did not directly reach. Clarifying the mechanism of the electron density enhancement in this altitude region is a subject which remains to be investigated further.

Acknowledgments. The sounding rocket experiment was conducted by the Institute of Space and Astronautical Science, Japan Aerospace Exploration Agency (ISAS/JAXA) as international project. We thank all members of the rocket experiments. The impedance probe was manufactured by System Keisoku Co., Ltd. The activities of the three authors (T. Ono, A. Kumamoto, and T. Suzuki) are supported by the Global COE Program "Global Education and Research Center for Earth and Planetary Dynamics" at Tohoku University.

\section{References}

Abdu, M. A., Outstanding problems in the equatorial ionospherethermosphere electrodynamics relevant to spread F, J. Atmos. Sol. Terr. Phys., 63, 869-884, 2001.
Balmain, K. G., The impedance of a short dipole antenna in a magnetoplasma, IEEE Trans. Ant. Prop., 12, 605-617, 1964.

Bernhardt, P. A., Three-dimensional time-dependent modeling of neutral gas diffusion in a nonuniform, chemically reactive atmosphere, J. Geophys. Res., 84, 793-802, 1979.

Bernhardt, P. A., Probing the magnetosphere using chemical releases from the CRESS satellite, Phys. Fluids B, 4, 2249-2256, 1992.

Heelis, R. A., Electrodynamics in the low and middle latitude ionosphere: a tutorial, J. Atmos. Sol. Terr. Phys., 66, 825-838, 2004.

Hunton, D. E., Long-term expansion characteristics of CRESS barium release clouds, Geophys. Res. Lett., 20, 563-566, 1993.

Lloyd, K. H. and G. Haerendel, Numerical modeling of the drift and deformation of ionospheric plasma clouds and of their interaction with other layers of the ionosphere, J. Geophys. Res., 78, 7389-7415, 1973.

Ma, T.-Z. and R. W. Schunk, Plasma cloud expansion in the ionosphere: Three-dimensional simulation, J. Geophys. Res., 96, 5793-5810, 1991.

Oya, H., Study on boundary value problems of magneto-active plasma and their applications to space observation, Ph.D. thesis, Kyoto University, Japan, 1966.

Oya, H. and T. Obayashi, Measurement of ionosphere electron density by a gyro-plasma probe: A rocket experiment by a new impedance probe, Rep. Ionos. Space Res. Jpn., 20, 199, 1966.

Rishbeth, H., The equatorial F-layer: progress and puzzles, Ann. Geophys., 18, 730-739, 2000.

Shiokawa, K., Y. Otsuka, C. Ihara, T. Ogawa, and F. J. Rich, Ground and satellite observations of nighttime medium-scale travelling ionospheric disturbance at midlatitude, J. Geophys. Res., 108, 1145, doi:10. 1029/2002JA009639, 2003.

Schunk, R. W. and E. P. Szuszczewicz, Plasma expansion characteristics of ionized clouds in the ionosphere: Macroscopic formulation, J. Geophys. Res., 96, 1337-1349, 1991.

Suzuki, T., T. Ono, J. Uemoto, M. Wakabayashi, T. Abe, A. Kumamoto, and M. Iizima, Sheath capacitance observed by impedance probes onboard sounding rockets: Its application to ionospheric plasma diagnostics, Earth Planets Space, 62, this issue, 579-587, 2010.

Szuszczewicz, E. P., D. E. Hunton, J. R. Wygant, and R. W. Schunk, Observations and model comparisons of early-time expansion characteristics of a satellite-borne barium-lithium release at F-region altitude, Geophys. Res. Lett., 20, 2031-2034, 1993.

Szuszczewicz, E. P., G. Earle, T. Bateman, Z. Klos, A. Kiraga, and R. W. Schunk, An "in situ" investigation of early time multi-ion expansion processes in an F region chemical release, J. Geophys. Res., 101, 1574915763, 1996.

Wakabayashi, M. and T. Ono, Electron density measurement under the influence of auroral precipitation and electron beam injection during the DELTA campaign, Earth Planets Space, 58, 1147-1154, 2006.

Wakabayashi, M., T. Ono, H. Mori, and P. A. Bernhardt, Electron density and plasma waves in mid-latitude sporadic-E layer observed during the SEEK-2 campaign, Ann. Geophys., 23, 2335-2345, 2005.

Watanabe, S., S. Nanbu, T. Abe, H. Habu, K. Ishisaka, T. Ono, Y. Otsuka, A. Saito, M. Yamamoto, and M.-Y. Yamamoto, WIND campaignRocket experiment for lithium release-, (in preparation).

Yamamoto, M.-Y., Y. Yokoyama, H. Habu, T. Abe, and S. Watanabe, Thermospheric neutral wind measurements by chemical release of Lithium, (in preparation).

J. Uemoto (e-mail: juemoto@nict.go.jp), T. Ono, T. Yamada, T. Suzuki, M.-Y. Yamamoto, S. Watanabe, A. Kumamoto, and M. Iizima 